\title{
SOLVABILITY OF HYPERBOLIC IBVPS THROUGH FILTERING*
}

\author{
DENIS SERRE ${ }^{\dagger}$
}

To Joel Smoller, with admiration.

\begin{abstract}
In a recent paper in collaboration with S. Benzoni, F. Rousset and K. Zumbrun, we uncovered the amazing fact that besides the classes of Hadamard unstable (no estimate at all) and of strongly stable hyperbolic IBVPs (estimates without loss of derivatives), there is a third "open" class, in the sense that it is stable under small disturbances of the coefficients (we apologize for the use of the word "stable" with two different meanings) in the PDEs and in the boundary conditions. We called the latter class (WR), because it has a Weak stability property (estimates with loss of one derivative) and because it is characterized by a "Real" characteristic set for the Lopatinsliı determinant.

We show here that with an appropriate filtering, systems in the class WR can be recast as strongly stable ones. It is remarkable that the same filtering is applied to both the solution and the data. Thus there is a hope to apply this linear theory to fixed point methods in non-linear problems, like the stability of two-dimensional vortex sheets when the jump in the velocity is larger than $2 \sqrt{2}$ times the sound speed.
\end{abstract}

Key words. Initial-boundary value problem, Hyperbolic PDE, Lopatinskiŭ condition

AMS subject classifications. 35L20 (primary), 35L50, 35L05

1. Introduction. We consider in this paper linear hyperbolic boundary value problems. We deal with operators having constant coefficients, while the domain $Q=\Omega \times \mathbb{R}$ is the half-space defined by $(x, t) \in \mathbb{R}^{d} \times \mathbb{R}$ with $x_{d}>0$. Recall that in this framework, estimates are obtained through the construction of a symbolic dissipative symmetrizer, together with the inversion of the Laplace-Fourier transform.

Let us take for definiteness a first-order operator

$$
L:=\partial_{t}+\sum_{\alpha=1}^{d} A^{\alpha} \partial_{\alpha},
$$

where $A^{1}, \ldots, A^{d}$ are real $n \times n$ matrices. In the sequel, we often denote $y=$ $\left(x_{1}, \ldots, x_{d-1}\right)$ the space variables along the boundary. We are given also a $p \times n$ real matrix $B$ of maximal rank $p$, and we consider the boundary value problem (BVP)

$$
\begin{aligned}
& L u=f, \quad x_{d}>0, \quad y \in \mathbb{R}^{d-1}, \quad t \in \mathbb{R}, \\
& B u=g, \quad x_{d}=0, \quad y \in \mathbb{R}^{d-1}, \quad t \in \mathbb{R} .
\end{aligned}
$$

Since we do not want to add several levels of difficulty, we shall assume that the boundary is non-characteristic, that is $A^{d} \in \mathbf{G L}_{n}(\mathbb{R})$. Likewise, we ask $L$ to be hyperbolic with characteristics of constant multiplicities, which means that the symbol

$$
A(\xi):=\sum_{\alpha} \xi_{\alpha} A^{\alpha}
$$

\footnotetext{
* Received June 22, 2005; accepted for publication December 9, 2005.

†UMPA (UMR 5669 CNRS), École Normale Supérieure de Lyon, 46, allée d'Italie, F-69364 Lyon, cedex 07, France (denis.serre@umpa.ens-lyon.fr). The research of the author was partially supported by the European IHP project "HYKE", contract \# HPRN-CT-2002-00282. It was achieved in part when the author was a Visiting Professor INdAM-GNAMPA at the Dipartimento di Matematica, Università degli studi di L'Aquila. The author is much indebted to his hosts D. Amadori, C. Lattanzio and P. Marcati.
} 
is diagonalisable for every $\xi \in \mathbb{R}^{d}$, with real eigenvalues of multiplicities not depending of $\xi \neq 0$. The latter hypothesis is fulfilled for instance in linearized gas dynamics, but not in MHD. It ensures that the pure Cauchy problem (in spatial domain $\mathbb{R}^{d}$ ) is strongly well-posed in $L^{2}$.

As is well-known, the theory is much more complicated for the BVP than for the Cauchy problem. Following Kreiss [10], we say that the BVP is strongly well-posed in $L^{2}$ if there exists a constant $C$ such that, for every $\gamma>0$ and every test function $u: \bar{\Omega} \rightarrow \mathbb{R}^{n}$, we have the estimate

$$
\operatorname{Out}_{\gamma}[u] \leq C \operatorname{In}_{\gamma}[u],
$$

where

$$
\begin{aligned}
\operatorname{Out}_{\gamma}[u] & :=\gamma \int_{\mathbb{R}} \int_{\Omega} e^{-2 \gamma t}|u(x, t)|^{2} d x d t+\int_{\mathbb{R}} \int_{\partial \Omega} e^{-2 \gamma t}|u(y, t)|^{2} d y d t, \\
\operatorname{In}_{\gamma}[u] & :=\frac{1}{\gamma} \int_{\mathbb{R}} \int_{\Omega} e^{-2 \gamma t}|L u(x, t)|^{2} d x d t+\int_{\mathbb{R}} \int_{\partial \Omega} e^{-2 \gamma t}|B u(y, t)|^{2} d y d t .
\end{aligned}
$$

In view of the potential applications to nonlinear problems such the stability of shock waves, it is crucial that in (4), we estimate the output $u$ and $\gamma_{0} u$ (its trace) in the same norms in which the input $L u$ and $B \gamma_{0} u$ are given.

Since the work by Kreiss (see also Sakamoto [12] for the theory of scalar equations of arbitrary order), it has been known that a necessary condition for strong wellposedness is the so-called Lopatinskiu condition. To describe it, we need to introduce the space $E_{i}(\tau, \eta)$ of incoming modes at frequencies $\tau \in \mathbb{C}$ (with $\Re \tau>0$ ) for the time variable and $\eta \in \mathbb{R}^{d-1}$ for the space variable. Taking the Fourier-Laplace transform of the homogeneous equation $L u=0$, we obtain the ODE

$$
\left(\tau I_{n}+i A(\eta)\right) v+A^{d} v^{\prime}=0 .
$$

The incoming modes are precisely the values of solutions $v$ that tend to zero as $x_{d} \rightarrow+\infty$. Thus $E_{i}(\tau, \eta)$ is nothing but the stable subspace of the matrix

$$
\mathcal{A}(\tau, \eta):=-\left(A^{d}\right)^{-1}\left(\tau I_{n}+i A(\eta)\right) .
$$

Since $L$ is hyperbolic, we know that this matrix has no purely imaginary eigenvalues. This implies that $E_{i}(\tau, \eta)$ depends analytically (and therefore holomorphically in $\tau$ ) on its arguments, and has constant dimension $p$, the number of positive eigenvalues of $A^{d}$ (we point out that it is the same $p$ than the number of scalar boundary conditions).

The restriction of $B$ to $E_{i}(\tau, \eta)$ is a linear map between two spaces of dimension $p$. We say that the Lopatinskiu condition is satisfied at point $(\tau, \eta)$ if this restriction is invertible. When it fails at some frequency pair, the BVP is strongly ill-posed in the Hadamard sense, see Hersh [8]. It is not hard to see that a necessary condition for strong well-posedness is that the Lopatinskil condition be satisfied uniformly in the sense that the inverse

$$
\left(\left.B\right|_{E_{i}(\tau, \eta)}\right)^{-1}
$$

exist and is bounded independently of $(\tau, \eta)$, as a linear map from $\mathbb{C}^{p}$ to $\mathbb{C}^{n}$. In other words, one needs a constant $C_{1}$ such that, for every $\tau$ with $\Re \tau>0$, for every $\eta \in \mathbb{R}^{d-1}$ and every $v \in E_{i}(\tau, \eta)$, we have

$$
|v| \leq C_{1}|B v|
$$


When $L$ has characteristics of constant multiplicities, Métivier has shown [11] that the uniform Lopatinskil condition (UKL) is also sufficient for strong well-posedness. See again Kreiss [10] for the strictly hyperbolic case. Within this framework, there is a practical way to check whether (UKL) holds or not. The constancy of multiplicities allows to extend the map $(\tau, \eta) \mapsto E_{i}(\tau, \eta)$ to non-zero boundary points $(\tau, \eta) \in$ $i \mathbb{R} \times \mathbb{R}^{d-1}$. We warn the reader that if $\tau \in i \mathbb{R}$, the space $E_{i}(\tau, \eta)$ contains the stable subspace of $\mathcal{A}(\tau, \eta)$, but the latter can be of dimension strictly less than $p$. This happens in particular when $\tau=i \rho$ and $\rho \gg|\eta|$, since then $\mathcal{A}(i \rho, \eta)$ has only pure imaginary eigenvalues. We also know that $E_{i}(i \rho, \eta)$ is contained in the central-stable subspace, but the latter may be too big. Therefore the determination of the extension is a delicate point in explicit computations. Within this framework, (UKL) amounts to saying that the Lopatinskil condition is satisfied at every non-zero point $(\tau, \eta)$ with $\Re \tau \geq 0$ and $\eta \in \mathbb{R}^{d-1}$.

1.1. Recalls about the WR class. For a few decades, a common belief was that problems satisfying the Lopatinskiu condition in a non-uniform way ${ }^{1}$ were nongeneric, in the sense that they could be converted in strongly ill/well-posed problems by slightly tuning their coefficients ; this "fact" was explicitly stated in [10]. Eventually the opposite was observed in [1], namely that these problems form a set of non-void interior. To explain what is going on, we need the notion of Lopatinskir determinant.

We begin with the observation that a basis $\left\{R_{1}(\tau, \eta), \ldots, R_{p}(\tau, \eta)\right\}$ can be chosen for the space $E_{i}(\tau, \eta)$, with the maximal regularity. In particular, each of the vector fields $R_{k}$ is analytic for $\Re \tau>0$ and continuous up to $\Re \tau=0$ if $E_{i}$ is (particularly if characteristics have constant multiplicities). Additionally, they can be chosen homogeneous of degree zero. The Lopatinskil condition at a point $(\tau, \eta)$ amounts to saying that $B R_{1}(\tau, \eta), \ldots, B R_{p}(\tau, \eta)$ are linearly independant. We therefore define the Lopatinskiu determinant by

$$
\Delta(\tau, \eta):=\operatorname{det}\left(B R_{1}(\tau, \eta), \ldots, B R_{p}(\tau, \eta)\right)
$$

Let $S^{+}$be the half-sphere defined by $\Re \tau \geq 0,|\tau|^{2}+|\eta|^{2}=1$. The Lopatinskiŭ condition at a given point $P$ means that $\Delta(P) \neq 0$, and (UKL) tells that $\Delta$ does not vanish for $(\tau, \eta) \in S^{+}$.

Let $\operatorname{char}(L)$ be the characteristic cone of $L$, defined as the set of pairs $(\rho, \xi) \in$ $\mathbb{R} \times \mathbb{R}^{d}$ such that $\operatorname{det}\left(\rho I_{n}+A(\xi)\right)=0$. The connected component $C^{+}$of $(1,0)$ in the complement of $\operatorname{char}(L)$ is called the forward cone of $L$. It turns out that $C^{+}$is convex, and every element of $C^{+}$is a direction of hyperbolicity of $L$ (see $[3,7,9]$ or [2], Chapter 1).

Let $\Gamma^{+}$be the image of $C^{+}$under the projection onto the $d$ first components:

$$
\Gamma^{+}=\pi C^{+}, \quad \pi\left(\rho, \eta, \xi_{d}\right)=(\rho, \eta) .
$$

This is a convex open cone called the hyperbolic component of the boundary frequencies. Then it is proved in [2], Chapter 9 , that for $(\rho, \eta) \in \Gamma_{+}$, the generalized stable subspace $E_{i}(i \rho, \eta)$ is of real type, meaning that it is spanned by a basis made of real vectors. It is therefore possible to choose the basis above in such a way that $R_{k}(i \rho, \eta)$ be real for every $k=1, \ldots, p$ and every $(\rho, \eta) \in \Gamma_{+}$. We notice also that within $\Gamma^{+}, E_{i}$ is actually analytic, although analyticity is lost at the boundary of $\Gamma^{+}$. Indeed analyticity is lost

\footnotetext{
${ }^{1}$ They form the complement of the two classes of strongly ill/well-posed problems.
} 
along an analytic set of the boundary $\Re \tau=0$ called the glancing set, which is defined as the set of singular values of the $\operatorname{map}^{2} \pi: \operatorname{char}(L) \rightarrow \mathbb{R} \times \mathbb{R}^{d-1}$.

An important consequence is that the restriction $D(\rho, \eta)$ of $\Delta$ to the hyperbolic boundary set $\Gamma^{+}$is a real analytic function. When $D$ vanishes in a non-degenerate way (namely $D=0$ together with $\nabla D=0$ has no solution), then its zero set is an analytic manifold ${ }^{3}$, isolated in the zero set of $\Delta$. Therefore the vanishing of $\Delta$ in $\Gamma^{+}$is compatible with the non-uniform Lopatinskil condition. Since non-degenerate analytic sets are stable under small analytic disturbances, we see that the class WR of systems for which $L$ has characteristics of constant multiplicities and such that $\Delta$ vanishes only on $\Gamma^{+}$, in a non-trivial and non-degenerate way, is open in the set of hyperbolic BVPs of constant multiplicities.

1.2. Filtering systems of WR class. Since (UKL) is a necessary condition for strong well-posedness, there is no hope that Estimate (4) holds true for systems of the class WR. Coulombel and Secchi $[4,5,6]$ have proved in various settings that an estimate with a loss of one derivative does hold. However, this loss of derivative creates difficulties when applying it to non-linear problems, where we have to iterate linear problems. Although there is a hope to overcome these difficulties through a use of Nash-Moser techniques, we think that it can be useful to have an analogue to (4), without any loss of derivative, to the price that both the data and the solutions are filtered with the same operators.

We see at least two motivations to this work. First of all, our estimates are more accurate than those of Coulombel and Secchi, in the sense that an $L^{2}$ data yields $L^{2}$ microlocal regularity (instead of $H^{-1}$ ) of the solution, except along some characteristic cone determined by the Lopatinskiu determinant. Additionally, it shows that certain components are microlocally in $L^{2}$ even around this characteristic cone: our estimate describes a polarisation phenomenon. At last, we do not really need that the data be $L^{2}$; it only needs to have the same kind of regularity as described above, that is $L^{2}$ off this characteristic cone, and some polarized form of the data being genuinely $L^{2}$.

Our goal in the forthcoming sections is therefore to show that an appropriate filtering of both the input and the output converts a BVP of class WR into a strongly well-posed BVP. We present first our strategy in the case of the wave equation and then develop it in the setting of first-order systems. It is remarkable that this strategy is partly successful also in the case of strongly ill-posed problem, yielding a new notion of "ghost solution" of the IBVP.

2. BVPs for the wave equation. We illustrate our strategy with the wave operator, and expect to adapt it to first-order operators in a future work. The fact that $L$ is scalar of second order is harmless, as the theories of Kreiss and Sakamoto [12] are essentially parallel. Besides, the wave operator is always a source of illuminating examples. It is also the only non-trivial scalar second-order hyperbolic operator, up to changes of coordinates.

Our boundary value problem is thus

$$
\begin{aligned}
L u:=\left(\partial_{t}^{2}-c^{2} \Delta_{x}\right) u=f, & x_{d}>0, \\
\partial_{\nu} u=\beta \partial_{t} u+v \cdot \nabla_{y} u+g, & x_{d}=0,
\end{aligned}
$$

where $c$ is the light speed and $\partial_{\nu}$ is the outer normal derivative, here $\partial_{\nu}=-\partial_{d}$.

\footnotetext{
${ }^{2}$ Note that we identify $\mathbb{R} \times \mathbb{R}^{d-1}$ with the boundary set of pairs $(\tau, \eta)$ such that $\Re \tau=0$, although the later is $i \mathbb{R} \times \mathbb{R}^{d-1}$.

${ }^{3}$ Actually, one proves in [2], Chapter 4 , that it is contained in an algebraic variety.
} 
Depending on the parameters $(\beta, v) \in \mathbb{R} \times \mathbb{R}^{d-1}$ the BVP is strongly ill/well-posed or weakly well-posed. Specifically, it is strongly well-posed when $\beta<0$ together with $|v|<|\beta| c$. If $d \geq 3$, it is strongly ill-posed when $\beta c \geq 1$, and of class WR in the interior of the complement of the two previous classes. If $d=2$, the strong illposedness concerns the parameters $(\beta, v)$ such that $1+|v|^{2}<\beta^{2} c^{2}$ with $\beta>0$; the class WR is again the interior of the complement of the two previous classes. See for instance [1] for calculations.

Our strategy is based on the following polynomial identity, where $\epsilon$ is a parameter to be chosen later:

(7) $\left(\epsilon^{2}-1\right)\left(Z^{2}-X^{2}\right)+4(X+Y)(Y+\epsilon Z)$

$$
=(2 Y+(1+\epsilon) X+(\epsilon-1) Z)(2 Y+(1-\epsilon) X+(\epsilon+1) Z) .
$$

In the context of the wave equation, we replace $X$ and $Z$ by $\partial_{d}$ and

$$
\Omega:=\omega\left(\partial_{t}, \frac{1}{i} \nabla_{y}\right), \quad \omega(\tau, \eta):=\sqrt{\frac{\tau^{2}}{c^{2}}+|\eta|^{2}}
$$

respectively. In the latter formula, the square root is the holomorphic determination of positive real part, defined in the complement of $(-\infty, 0]$. The function $\omega$ is welldefined and analytic for $\eta \in \mathbb{R}^{d-1}$ and $\Re \tau>0$, and can be extended by continuity to $(\tau, \eta) \in i \mathbb{R} \times \mathbb{R}^{d-1}$. With this choice, we have $Z^{2}-X^{2}=c^{-2} L$. Then $Y$ is the part of the boundary operator $B$ that acts tangentially to the frontier: $Y=\beta \partial_{t}+v \cdot \nabla_{y}$, so that $B=X+Y$. Because of (7), we introduce the following operators

$$
\begin{aligned}
& R:=Y+\epsilon Z=\beta \partial_{t}+v \cdot \nabla_{y}+\epsilon \Omega, \\
& P:=2 Y+(1+\epsilon) X+(\epsilon-1) Z=(1+\epsilon) \partial_{d}+(\epsilon-1) \Omega+2 Y, \\
& E:=2 Y+(1-\epsilon) X+(\epsilon+1) Z=(1-\epsilon) \partial_{d}+(1+\epsilon) \Omega+2 Y .
\end{aligned}
$$

The fact that the operator $R$ acts only in the variables $(t, y)$, but not in $x_{d}$, will allow us to apply it to functions defined on the physical boundary $x_{d}=0$.

For the moment, we rewrite (7) as

$$
\frac{\epsilon^{2}-1}{c^{2}} L+4 B R=P E
$$

and introduce an auxiliary unknown, using the operator $P$ as a filter:

$$
z:=P u \text {. }
$$

We point out that when $\beta \in[0,1 / c)$, the symbol of $P$ vanishes at those boundary points where the Lopatinskil condition is violated. Therefore $z$ does not see the singularities that are propagated in $u$.

Applying $P$ to the wave equation, we see that $z$ obeys to a wave equation with a filtered data:

$$
L z=P f, \quad(t, x) \in Q .
$$

Of course, we are not allowed to apply $P$ to the boundary condition (6) since $P$ contains an $x_{d}$-derivative. However, we may apply $R$, yielding

$$
B R u+R g=0 .
$$


We now use (11) to convert (13) into a boundary condition for $z$ :

$$
E z=\frac{\epsilon^{2}-1}{c^{2}} f-4 R g, \quad(t, x) \in \partial Q .
$$

At this stage, we have decomposed our boundary value problem $(5,6)$ into two sub-problems. In the first one, we construct the auxiliary unknown $z$ by solving the BVP $(12,14)$, while in the second one we reconstruct $u$ through

$$
P u=z, \quad(t, x) \in Q,
$$

We point out that the trace of $f$ along the boundary is involved in the boundary condition (14).

Our expectation is to find an $\epsilon \in \mathbb{R}$ such that both $(12,14)$ and $(15)$ be strongly stable BVPs. For $(5,6)$, this means the (UKL) condition. Since $P$ is a homogeneous first order $\Psi D O$, of the form $\partial_{d}+Q\left(\partial_{t}, \nabla_{y}\right)$, the boundary is non-characteristic; we thus ask that the Cauchy problem be well-posed for $P$, and that the waves be outgoing, which implies that the BVP with no boundary condition is strongly well-posed.

Theorem 2.1. Assume that $|v|<\beta c<1 / 2$. Then there exists an $\epsilon$ such that both $(12,14)$ and (15) are strongly stable BVPs. In particular, the $B V P(5,6)$ is uniquely solvable when $\operatorname{Pf} \in L_{\gamma}^{2}(Q)$ and $g \in L_{\gamma}^{2}(\partial Q)$, where $Q \in \mathbb{R}_{t} \times \mathbb{R}_{x}^{d}$ is defined by $x_{d}>0$.

There holds the following estimates:

$$
\begin{aligned}
\gamma\left\|\nabla_{x, t} P u\right\|_{L_{\gamma}^{2}(Q)}^{2}+\left\|\nabla_{x, t} P u\right\|_{L_{\gamma}^{2}(\partial Q)}^{2} & \leq C\left(\frac{1}{\gamma}\|P f\|_{L_{\gamma}^{2}(Q)}^{2}+\|R g\|_{L_{\gamma}^{2}(\partial Q)}^{2}\right), \\
\gamma\left\|\nabla_{x, t} u\right\|_{L_{\gamma}^{2}(Q)}^{2}+\left\|\nabla_{x, t} u\right\|_{L_{\gamma}^{2}(\partial Q)}^{2} & \leq \frac{C}{\gamma^{2}}\left(\frac{1}{\gamma}\|P f\|_{L_{\gamma}^{2}(Q)}^{2}+\|R g\|_{L_{\gamma}^{2}(\partial Q)}^{2}\right) .
\end{aligned}
$$

\section{REMARKS.}

- In the above result, we may take any $\epsilon \in(0,1-2 \beta c)$.

- In (17), we estimate $\nabla_{x, t} u$ in terms of $P f$ and $R g$ instead of $f$ and $g$. Since $P$ and $R$ are first-order, we have a loss of one derivative, compared to the strongly stable case. However, asking $\operatorname{Pf} \in L_{\gamma}^{2}(Q)$ is clearly a weaker assumption than asking $f \in \dot{H}_{\gamma}^{1}(Q)$, since the symbol of $P$ vanishes along a cone. Likewise, the assumption that $R g \in L_{\gamma}^{2}(\partial Q)$ is weaker than $g \in \dot{H}_{\gamma}^{1}(\partial Q)$. Notice that the loss of one derivative is associated to an extra factor $\gamma^{-2}$, which preserves the homogeneity in the estimates.

- The solution $u$ that is constructed in the above procedure does satisfy the original BVP $(5,6)$. On the one hand $(12)$ and $(15)$ give $P(L u-f)=0$, whence $L u=f$ since $P$ is injective on $H_{\gamma}^{s}(Q)$. On the other hand, (13) and (11) yield $R(B u+g)=0$, which gives $B u+g=0$ because $R$ is injective (this will be proved below) on $H_{\gamma}^{s}(\partial Q)$.

Proof.

UKL for $(\mathbf{1 2}, \mathbf{1 4})$. Since the wave operator is hyperbolic with characteristics of constant multiplicities, and since the boundary is not characteristic (there is a $\partial_{d}^{2}$ in $L$ ), we only need to check that (UKL) holds true. This means verifying that the Lopatinskiu determinant ${ }^{4} \Delta(\tau, \eta)$ does not vanish for $\Re \tau \geq 0$.

\footnotetext{
${ }^{4}$ We apologize for the multiple use of the letter $\Delta$, hoping that it will not be confusing.
} 
The function $\Delta$ is obtained by taking the symbol of the boundary operator $E$, while replacing $\partial_{d}$ by $-\omega(\tau, \eta)$, since incoming waves at Laplace-Fourier frequencies $(\tau, \eta)$ correspond to solutions of

$$
\partial_{d} Z=-\omega(\tau, \eta) Z
$$

Thus we have

$$
\Delta(\tau, \eta)=2(\beta \tau+i v \cdot \eta+\epsilon \omega(\tau, \eta)) .
$$

This is precisely the Lopatinskiu determinant for $\left(L, Y-\epsilon \partial_{d}\right)$. From [1], we know that (UKL) holds true if, and only if,

$$
\beta \epsilon>0 \text { and }|v|<|\beta| c .
$$

Cauchy problem for $P$. The symbol of $P$ is $i(1+\epsilon) \xi+(\epsilon-1) \omega(\tau, \eta)+2(\beta \tau+i v \cdot \eta)$, where $\xi$ is the variable dual to $x_{d}$. We have to check that given $(\eta, \xi) \in \mathbb{R}^{d}$ and $\Re \tau>0$, the symbol does not vanish. Since $\xi$ is an arbitrary real number, this means that

$$
\Re((\epsilon-1) \omega(\tau, \eta)+2(\beta \tau+i v \cdot \eta)) \neq 0,
$$

namely

$$
\Re((\epsilon-1) \omega(\tau, \eta)+2 \beta \tau) \neq 0
$$

for every pair $(\tau, \eta)$. We easily find the equivalent condition that

$$
\frac{\epsilon-1}{\beta} \notin[-c, 0] \text {. }
$$

BVP for $P$. Since $P=\partial_{d}+\cdots$, the boundary is non-characteristc for the first order operator $P$. Since $P$ is scalar, it displays either outgoing waves at every frequency $(\tau, \eta)$, or incoming waves. From Laplace-Fourier transform and PaleyWiener theorem, the BVP (15) with no boundary condition is strongly well-posed in $H_{\gamma}^{s}$ provided the waves are outgoing. Since the incoming/outgoing character does not depend on the frequency, it is enough to consider waves with frequency $(\tau, \eta=0)$. These are governed by the transport operator

$$
P_{0}=(1+\epsilon) \partial_{d}+((\epsilon-1) / c+2 \beta) \partial_{t},
$$

whose wave velocity is

$$
c(1+\epsilon) /(\epsilon-1+2 \beta c) .
$$

We therefore find the condition

$$
(1+\epsilon)(\epsilon-1+2 \beta c)<0 .
$$

Uniqueness for $R$ in $H_{\gamma}^{s}(\partial Q)$. Here, we only need that the symbol $r(\tau, \eta):=$ $\beta \tau+i v \cdot \eta+\epsilon \omega(\tau, \eta)$ does not vanish for $\eta \in \mathbb{R}^{d-1}$ and $\Re \tau \neq 0$. This is clearly true at least when $\beta \epsilon>0$ (take the real part of $r$ ). Since this inequality is implied by (18), we do not push forward our analysis. 
Choice of $\epsilon$. We match now Conditions $(18,19,20)$. Since $\beta$ is positive, we must have $\epsilon>0$, whence $\epsilon<1-2 \beta c$. Since $1-2 \beta c<1-\beta c$, (19) is automatically satisfied. Hence any $\epsilon \in(0,1-2 \beta c)$ works.

Estimates. With $\epsilon \in(0,1-2 \beta c)$, both BVPs are strongly well-posed. For (15), we have an estimate

$$
\gamma\|w\|_{L_{\gamma}^{2}(Q)}^{2}+\|w\|_{L_{\gamma}^{2}(\partial Q)}^{2} \leq \frac{C}{\gamma}\|P w\|_{L_{\gamma}^{2}(Q)}^{2} .
$$

From the (UKL) property of $(12,14)$, we have the estimate

$$
\gamma\left\|\nabla_{x, t} z\right\|_{L_{\gamma}^{2}(Q)}^{2}+\left\|\nabla_{x, t} z\right\|_{L_{\gamma}^{2}(\partial Q)}^{2} \leq C\left(\frac{1}{\gamma}\|P f\|_{L_{\gamma}^{2}(Q)}^{2}+\left\|\left(\epsilon^{2}-1\right) f-4 R g\right\|_{L_{\gamma}^{2}(\partial Q)}^{2}\right) .
$$

Combining this inequality with (21) applied to $w=f$, we obtain (16) since $z=P u$. Then (21) applied to $w=u$, together with (16), give (17).

REMARKS.

- It is remarkable that the first step works even if $\beta c \geq 1 / 2$, although the original BVP is strongly ill-posed in the case $\beta c \geq 1$ ! In particular, there exists always a "ghost solution" $z$, even in cases where $u$ cannot be recovered. This reveals that every bad or good feature is concentrated within Problem (15).

- When $1 / 2 \leq \beta c<1$, we can chose $\epsilon$ in such a way that $(12,14)$ is strongly well-posed, as well as the Cauchy problem for $P$, but then the waves of $P$ are ingoing. To solve a BVP for $P$, we need a boundary data $u=u_{0}$ on $\partial Q$. This data is obtained by eliminating $\partial_{d} u$ between (15) and (6) to obtain

$$
(\Omega-Y) u=\frac{z+(\epsilon+1) g}{\epsilon-1} .
$$

This is an evolution equation along $\partial Q$, which turns out to be uniquely solvable. As a matter of fact, the operator $\Omega-Y$ is a first-order factor of

$$
\mathcal{L}:=\Omega^{2}-Y^{2}=\frac{1}{c^{2}} \partial_{t}^{2}-\Delta_{y}-\left(\beta \partial_{t}+v \cdot \nabla_{y}\right)^{2} .
$$

This operator is wave-like, with a forward cone containing the characteristic cone of $L$. In other words, the waves travel faster under $\mathcal{L}$ than under $\partial_{t}^{2}-$ $c^{2} \Delta_{y}$. For instance, if $v=0$, then

$$
\mathcal{L}=\partial_{t}^{2}-\tilde{c}^{2} \Delta_{y}
$$

where the boundary velocity $\tilde{c}$ is given by

$$
\tilde{c}:=\frac{c}{\sqrt{1-\beta^{2} c^{2}}},
$$

which is larger than the light speed!

Notice that in this case, the norm of $\left.f\right|_{\partial Q}$ in $L_{\gamma}^{2}$ cannot be estimated from that of $P f$. Therefore the estimates of Theorem 2.1 are not valid or at least have not been proved so far. We may state nonetheless that

$$
\gamma\left\|\nabla_{x, t} z\right\|_{L_{\gamma}^{2}(Q)}^{2}+\left\|\nabla_{x, t} z\right\|_{L_{\gamma}^{2}(\partial Q)}^{2} \leq C\left(\frac{1}{\gamma}\|P f\|_{L_{\gamma}^{2}(Q)}^{2}+\|f\|_{L_{\gamma}^{2}(\partial Q)}^{2}+\|R g\|_{L_{\gamma}^{2}(\partial Q)}^{2}\right)
$$


and

$$
\gamma\left\|\nabla_{x, t} u\right\|_{L_{\gamma}^{2}(Q)}^{2} \leq \frac{C}{\gamma^{2}}\left(\frac{1}{\gamma}\|P f\|_{L_{\gamma}^{2}(Q)}^{2}+\|f\|_{L_{\gamma}^{2}(\partial Q)}^{2}+\|R g\|_{L_{\gamma}^{2}(\partial Q)}^{2}\right) .
$$

- Since $\Omega-Y$ is an operator of principal type with a non-trivial characteristic cone when $\beta \in(0,1 / c)$, there are non-trivial solutions of $(\Omega-Y) \phi=0$. Such $\phi$ 's are solutions of the wave equation along the boundary, with wave velocity $\tilde{c}$. This observation is not incompatible with the uniqueness result in weighted spaces $\dot{H}_{\gamma}^{s}$ because this wave equation preserves the energy and therefore the only solution whose (constant) energy is in $L^{1}\left(e^{-2 \gamma t} d t\right)$ for some $\gamma>0$, is the trivial one.

- In both $(12,14)$ and $(15)$, the solution obeys to the causality principle, which tells that its restriction to times $t \in(-\infty, T)$ depends only on the restriction of the data on the same interval. This is due to the analyticity of the symbols in the half-space $\Re \tau>0$ and to the Paley-Wiener Theorem. In particular, it is possible to solve the initial-boundary value problem (IBVP) with homogeneous data $u(0, x) \equiv 0$. However, we do not exclude that the general IBVP suffers an additional loss of regularity in the WR case.

3. Variable coefficients, general domains. The procedure described in the previous section adapts easily to BVPs for general second order scalar hyperbolic operators, with smooth variable coefficients. In particular, it is efficient in solving noncharacteristic BVPs in smooth bounded domains.

After localisation and change of variables, we may assume that the domain $Q$ is defined as above by $x_{d}>0$. Up to a multiplication by a smooth non-vanishing function, we may assume that $L$ writes

$$
L=-\partial_{d}^{2}+\cdots
$$

where the remainder has variable coefficients. The principal symbol, a quadratic form, can be rewriten as

$$
\ell=\left(\xi_{d}+w(t, x) \cdot \eta+w_{0}(t, x) \xi_{0}\right)^{2}-Q\left(\xi_{0}, \eta ; t, x\right),
$$

where $Q(\cdot, \cdot ; t, x)$ is a non-degenerate quadratic form of signature $(1, d-1)$. When $\Re \tau \neq 0, Q(-i \tau, \eta ; t, x) \notin(-\infty, 0]$. We wish to apply the identity (7) with

$$
X=\partial_{d}+w(t, x) \cdot \nabla_{y}+w_{0}(t, x) \partial_{t}, \quad Z:=\mathrm{Op}(\Omega), \quad Y\left(\partial_{t}, \nabla_{y}\right):=B-X,
$$

where $B=\partial_{d}+\cdots$ is the boundary operator and $\Omega(\tau=i \rho, \eta ; t, x):=\sqrt{Q}$ is defined for $\Re \tau>0$ as the square root of positive real part, and by continuity along $\Re \tau=0$. We point out that since $\Omega$ is analytic in $\tau$ as $\Re \tau>0$, the definition of $Z$ is unambiguous in spite of variable coefficients. Since $\Omega$ has singularities along $\Re \tau=0$, we begin by defining a one-parameter family of operators

$$
Z_{\gamma}:=\mathrm{Op}_{t, y}(\Omega(\gamma+i \cdot, \cdot ; t, x)) .
$$

Because of holomorphy, we have $Z_{\gamma}=e_{-\gamma} Z e_{\gamma}$ for some operator $Z$, where $e_{\gamma}$ is the multiplication by $\exp (\gamma t)$.

We now define operators $R, P$ and $E$ as in $(8,9,10)$. Because of variable coefficients, the identity (11) is true up to a reminder of order one $r$ :

$$
\left(\epsilon^{2}-1\right) L+4 R B=E P+r .
$$


Notice that since $Y, Z, Q$ and $X-\partial_{d}$ are operators in the variables $(y, t)$ only, $r=r\left(\partial_{t}, \nabla_{y}\right)$ does not act on the $x_{d}$ variable.

With (23), we can embed the problem

$$
L u=f \quad\left(x_{d}>0\right), \quad B u=g \quad\left(x_{d}=0\right)
$$

into the following coupled BVP

$$
\begin{aligned}
L z+a u & =P f \quad\left(x_{d}>0\right), \\
P u & =z \quad\left(x_{d}>0\right), \\
E z+r u & =\left(\epsilon^{2}-1\right) f+4 R g \quad\left(x_{d}=0\right),
\end{aligned}
$$

where $a$ is pseudo-differential of first order.

The principal part in System $(28,26,30)$ is obtained by dropping the operators $a$ and $r$. When the assumption of Theorem 2.1 is satisfied pointwisely, a symbolic symmetrizer can be constructed, which depends smoothly on its arguments. Thus strong stability holds for the system

$$
\begin{aligned}
& L z=F \quad\left(x_{d}>0\right), \\
& P u=z \quad\left(x_{d}>0\right), \\
& E z=G .
\end{aligned}
$$

Then the full sytem $(25,26,27)$ can be analyzed by means of the Duhamel formula, treating the lower order terms $a u$ and $r u$ as source terms and iterating in $L_{\gamma}^{2}$. This iteration is a contraction provided $\gamma>0$ is large enough. At the end, we obtain the well-posedness of the BVP (24) in the same filtered terms as in the constant coefficients case.

4. Filtering first-order systems of class WR. We go back to first order BVPs $(2,3)$, with a hyperbolic operator $L$ of the form (1), for which the boundary is not characteristic. We assume that its characteristics have constant multiplicities. Being interested in estimates, we work directly with the Laplace-Fourier counterpart

$$
\begin{gathered}
\left(\tau I_{n}+i A(\eta)\right) v+A^{d} v^{\prime}=f, \quad x_{d}>0, \\
B v(0)=g .
\end{gathered}
$$

We assume that the Lopatinskiu condition is satisfied everywhere in $\Re \tau \geq 0$ but along an algebraic subset $\Lambda$ of the hyperbolic part $\Gamma^{+}$of the boundary frequencies. We assume in fact that the Lopatinskiu determinant $\Delta$ vanishes only at first order along $\Lambda$; in particular, $\Lambda$ is a compact smooth real variety of codimension one in $\Gamma^{+}$.

The micro-local counterpart of (4) is an estimate of the form

$$
\gamma \int_{0}^{+\infty}|v|^{2} d x_{d}+|v(0)|^{2} \leq C\left(\frac{1}{\gamma} \int_{0}^{+\infty}|f|^{2} d x_{d}+|g|^{2}\right)
$$

for the $L^{2}$ solutions of $(31,32)$, where $C$ does not depend either on the data $(f, g)$ or the parameter $(\tau, \eta)$ (here $\gamma:=\Re \tau>0$ ). Because $L$ has characteristics of constant multiplicities, we know (see $[11,2])$ that $(33)$ near a given $\left(\tau_{0}, \eta_{0}\right)$ is equivalent to the fact that the Lopatinskil condition $\Delta\left(\tau_{0}, \eta_{0}\right)$ is satisfied. Therefore (33) holds true, except when we approach $\Lambda$. Actually, we could write (33), with a constant $C$ that depends on $(\tau, \eta)$ and blows up as the distance from $(\tau, \eta)$ to $\Lambda$ tends to zero. 
We now restrict to points in a neighbourhood of $\Lambda$. In particular, $(\tau, \eta)$ keeps away from the glancing set and we can project on $E_{i}(\tau, \eta)$ and on the outgoing space $E_{o}(\tau, \eta)$ in a smooth way. We call $\pi_{i, o}(\tau, \eta)$ the corresponding projections. There holds $\pi_{i}+\pi_{o}=I_{n}$. Each of $\pi_{i, o}$ depends analytically on $(\tau, \eta)$ as long as we keep away from the glancing set. From now on, we decompose $v$ into incoming/outgoing modes $v_{i, o}:=\pi_{i, o} v$. We point out that such a decomposition of $f$ must be made only after multiplying by $\left(A^{d}\right)^{-1}$; we thus define $f_{i, o}:=\pi_{i, o}\left(A^{d}\right)^{-1} f$.

We then rewrite ( 31 in the following form, using projections:

$$
\begin{aligned}
v_{i}^{\prime} & =\mathcal{A}_{i}(\tau, \eta) v_{i}+f_{i}, \\
v_{o}^{\prime} & =\mathcal{A}_{o}(\tau, \eta) v_{o}+f_{o},
\end{aligned}
$$

where $\mathcal{A}_{i, o}(\tau, \eta)$ is the restriction of $\mathcal{A}(\tau, \eta)$ to its stable/unstable subspace $E_{i, o}(\tau, \eta)$. Here the data $f_{i, o}$ are square integrable, and we look for solutions $v_{i, o}$ with the same property. It is well-known that such a $v_{o}$ is uniquely determined by the ODE (no need of a boundary condition here) through the Duhamel formula

$$
v_{o}(x)=-\int_{x}^{+\infty}\left(\exp \left((x-z) \mathcal{A}_{o}\right)\right) f_{o}(z) d z .
$$

However, the square-integrable solutions of (34) form a large family, given by the formula

$$
v_{i}(x)=\left(\exp \left(x \mathcal{A}_{i}\right)\right) v_{i}(0)+\int_{0}^{x}\left(\exp \left((x-z) \mathcal{A}_{i}\right)\right) f_{i}(z) d z
$$

where $v_{i}(0)$ must be determined through the boundary condition (32). Specifically, we have to solve

$$
B v_{i}(0)=g+B \int_{0}^{+\infty}\left(\exp \left(-z \mathcal{A}_{o}\right)\right) f_{o}(z) d z .
$$

Since the operator $B: E_{i}(\tau, \eta) \rightarrow \mathbb{C}^{p}$ remains uniformly bounded, its inverse is bounded by a constant times $|\Delta(\tau, \eta)|^{-1}$, a quantity that blows up precisely on $\Lambda$. We therefore have

$$
\left|v_{i}(0)\right| \leq \frac{C}{|\Delta|}\left(|g|+\int_{0}^{+\infty}\left|\left(\exp \left(-z \mathcal{A}_{o}\right)\right) f_{o}(z)\right| d z\right) .
$$

Let $\left.K_{o}(\tau, \eta)\right)$ be the kernel defined as $\exp \left(-z \mathcal{A}_{o}\right)$ if $z>0$ and 0 otherwise. Likewise, we define the kernel $K_{i}(\tau, \eta)$ as $\exp \left(-z \mathcal{A}_{i}\right)$ if $z<0$ and 0 otherwise. From CauchySchwarz and (39), we have

$$
\left|v_{i}(0)\right| \leq \frac{C}{|\Delta|}\left(|g|+\left\|K_{o}\right\|_{2}\left\|f_{o}\right\|_{2}\right),
$$

where $\|\cdot\|_{2}$ stands for the $L^{2}$-norm on $\mathbb{R}^{+}$. Since (36) and (37) involve convolution products, we also have

$$
\left\|v_{o}\right\|_{2} \leq\left\|K_{o}\right\|_{1}\left\|f_{o}\right\|_{2}, \quad\left\|v_{i}\right\|_{2} \leq\left\|K_{i}\right\|_{2}\left|v_{i}(0)\right|+\left\|K_{i}\right\|_{1}\left\|f_{i}\right\|_{2} .
$$

These inequalities are completed by the obvious following bounds, which are optimal near $\Gamma^{+}$:

$$
\left\|K_{i, o}\right\|_{1} \leq \frac{C}{\gamma}, \quad\left\|K_{i, o}\right\|_{2} \leq \frac{C}{\sqrt{\gamma}}
$$


Hence we find

$$
\left\|v_{o}\right\|_{2} \leq \frac{C}{\gamma}\left\|f_{o}\right\|_{2}, \quad\left\|v_{i}\right\|_{2} \leq \frac{C}{\gamma}\left(\frac{\left\|f_{o}\right\|_{2}+\gamma^{1 / 2}|g|}{|\Delta|}+\left\|f_{i}\right\|_{2}\right) .
$$

This, together with (40), clearly show that only the incoming modes pay the loss of uniformity in the Lopatinskiu condition. This suggest to introduce a filtering symbol $p(\tau, \eta)$ defined (at least in a neighbourhood of $\Gamma^{+}$) as

$$
p(\tau, \eta):=\pi_{o}(\tau, \eta)+\Delta \pi_{i}(\tau, \eta) .
$$

With this operator in hands, we have the estimate

$$
\|p v\|_{2} \leq \frac{C}{\gamma}\left(\left\|f_{o}\right\|_{2}+\gamma^{1 / 2}|g|+|\Delta|\left\|f_{i}\right\|_{2}\right),
$$

or in other words

$$
\|p v\|_{2} \leq \frac{C}{\gamma}\left\|p\left(A^{d}\right)^{-1} f\right\|_{2}+\frac{C}{\sqrt{\gamma}}|g| .
$$

We emphasize that (43) is precisely, up to the boundary terms, the estimate (33) where $v$ and $f$ are replaced by filtered solution $p v$ and filtered data $p\left(A^{d}\right)^{-1} f$. Likewise we have

$$
|p v(0)| \leq \frac{C}{\sqrt{\gamma}}\left\|p\left(A^{d}\right)^{-1} f\right\|_{2}+|g|
$$

which is again the adaptation of (33) with filtered data and solution.

The definition (42) makes $p$ the symbol of a pseudo-differential operator of zero order, which is micro-locally elliptic, except along $\Lambda$. It might be singular, and even unbounded, along the glancing set since the projectors blow up there. However this singularity is irrelevant since we know that Estimate (33) does hold near the glancing set. Therefore we feel free to smooth out the symbol $p$ along the glancing set, in such a way that $p$ be $\mathcal{C}^{\infty}$, homogeneous of degree zero, and its characteristic cone be $\Lambda$ where it vanishes only at first order.

4.1. The analytic case. Let us assume for the moment that we do not need to smooth out the symbol $p$ near the glancing set. This will be case whenever $\Delta$ can be $\operatorname{chosen}^{5}$ in such a way that $\Delta \equiv 1$ along the glancing set ${ }^{6}$. As a matter of fact, the formula

$$
p(\tau, \eta)=I_{n}+(\Delta(\tau, \eta)-1) \pi_{i}(\tau, \eta)
$$

shows that $p$ remains bounded because, the scalar factor cancels the singularity of the projector.

In this analytic case, let us define a pseudo-differential operator $P:=p\left(\partial_{t},-i \nabla_{y}\right)$ from the trace of the symbol $p$ on $\Re \tau=0$. Then analyticity tells that

$$
P_{\gamma}:=p\left(\gamma+\partial_{t},-i \nabla_{y}\right)=e^{\gamma t} P e^{\gamma t} .
$$

\footnotetext{
${ }^{5}$ Recall that $\Delta$ is defined only up to an analytic non-vanishing factor.

${ }^{6}$ This amounts to prescribing values of an analytic function on some analytic varieties of codimension one in the boundary.
} 
Hence our microlocal estimates exactly mean that (4) holds true with

$$
\begin{aligned}
\operatorname{Out}_{\gamma}[u] & :=\gamma \int_{\mathbb{R}} \int_{\Omega} e^{-2 \gamma t}|P[u(x, t)]|^{2} d x d t+\int_{\mathbb{R}} \int_{\partial \Omega} e^{-2 \gamma t}|P[u(y, t)]|^{2} d y d t, \\
\operatorname{In}_{\gamma}[u] & :=\frac{1}{\gamma} \int_{\mathbb{R}} \int_{\Omega} e^{-2 \gamma t}\left|P\left[\left(A^{d}\right)^{-1} L u(x, t)\right]\right|^{2} d x d t+\int_{\mathbb{R}} \int_{\partial \Omega} e^{-2 \gamma t}|B u(y, t)|^{2} d y d t .
\end{aligned}
$$

We point out that the data comes out through the operator

$$
\tilde{L}:=\left(A^{d}\right)^{-1} L=\frac{\partial}{\partial x_{d}}+\cdots,
$$

which seems to be a canonical form for a first-order BVP with a non-characteristic boundary.

We also notice that because of the analyticity of the symbol in the whole half-space $\Re \tau>0$, and thanks to the Paley-Wiener Theorem, $P$ has the causality property.

The role of the "filtered" estimate (33) is, as in Section 2, to provide an existence and uniqueness result for the auxiliary unknown $z:=P u$, when $\tilde{f}:=\tilde{L} u$ is given such that $P \tilde{f} \in L_{\gamma}^{2}$ and $g \in L_{\gamma}^{2}$.

4.2. Reconstruction. From $z$, which is known to exist in $L_{\gamma}^{2}$, we have to reconstruct the solution $u$ from the equation

$$
P u=z .
$$

This is a $\psi$-Differential problem with a constant coefficients operator that acts on $(t, y)$ only. Here $x_{d}$ could be considered as a parameter. A typical estimate is

$$
\|u\|_{L_{\gamma}^{2}(Q)} \leq \frac{C}{\gamma}\left\|\nabla_{y, t} z\right\|_{L_{\gamma}^{2}(Q)},
$$

the loss of one derivative being due to the order zero of $P$.

Since $P$ is an operator of principal type with a known smooth characteristic type, it acts as a hyperbolic operator, except that it is zero order.

Acknowledgment.. This work was boosted by a conversation with Paolo Secchi. I am grateful to him for his questions and his interest in the topic.

\section{REFERENCES}

[1] S. Benzoni-Gavage, F. Rousset, D. Serre, K. Zumbrun, Generic types and transitions in hyperbolic initial-boundary value problems, Proc. Royal Soc. Edinburgh, A, 132 (2002), pp. 1073-1104.

[2] S. Benzoni-Gavage, D. Serre, Multi-dimensional hyperbolic partial differential equations: First-order systems and applications, Oxford University Press, Oxford, to appear (2006).

[3] J. Chazarain, A. Piriou, Introduction to the theory of linear partial differential equations, North-Holland, Amsterdam (1982).

[4] J.-F. Coulombel, Weak stability of nonuniformly stable multidimensional shocks, SIAM J. Math. Anal., 34 (2002), pp. 401-443.

[5] J.-F. Coulombel, Weakly stable multidimensional shocks, Ann. Inst. Henri Poincaré Anal. Non Linéaire, 21 (2004), pp. 142-172.

[6] J.-F. Coulombel, P. SecCHI, The stability of compressible vortex sheets in two space dimensions, Indiana Univ. Math. J., 53 (2004), pp. 941-1012.

[7] L. GÅRING, Linear hyperbolic partial differential equations with constant coefficients, Acta Math., 85 (1951), pp. 1-62; An inequality for hyperbolic polynomials, J. Math. Mech., 8 (1959), pp. 957-965.

[8] R. Hersh, Mixed problems in several variables, J. Math. Mech., 12 (1963), pp. 317-334. 
[9] L. HÖRMANDER, Lectures on nonlinear hyperbolic differential equations, Springer-Verlag, Berlin (1997).

[10] H.-O. KreISs, Initial boundary value problems for hyperbolic systems, Comm. Pure Appl. Math., 23 (1970), pp. 277-298.

[11] G. MÉTIVIER, The block structure condition for symmetric hyperbolic systems, Bull. London Math. Soc., 32 (2000), pp. 689-702.

[12] R. SAкамото, Hyperbolic boundary value problems, Cambridge University Press, Cambridge (1982). 